le foie est alors fréquemment envahi par les formes larvaires, Cercaires si caractéristiques, de Sanguinicola. Mais il ret bien entendu que ce seul examen des Mollusques, s'il est néfalif, n'est pas suffisant pour affirmer l'absence de la maladie chez les Poissons de l'étang.

\title{
LA TAXE DE LUXE ET LA PISCIGULTURE
}

\author{
Par M. Chanles GUyOT . \\ Ancien Directeur de l'Ecole Nationale des Eaux th Forèts.
}

Ies lois des 3 r juillet r 920 et 13 juillet $19 x$ ont instilué une taxe de Juxe sur les baux de pèche et de chisse yui sobleve, actuellement, à douze four cent ( I $_{2} \%$ ) du monlaml du lower. II con résulte une charge fort lourde pour les pisciculteurs qui m: sont pis propriéfilires, mais amodiataires des fonds d'eau qu'jls cxploilcnl.

Ceux qui élèvent la Carpe dans les élanges ou la Truite dans les viviers ne le font pas, sauf rare exception, lans le hut volupluaire, mais utilitaire. Ceux qui ont loué ces étanģs ou viviers paraissent bien assimilables aux fermiers ruraux et le loisson ru ils oluienuent n'cst rien autre chose "lu'un bétail d'eau. It semblerait donc normal quiils fussent exempts de Is laxe à laquelle, depuis ure dizaine d'années, le législateur a assujetti les locations de pêche. Il n'en ra pas ainsi cependant, ce qui a provoqué maintes protestations des intéresés el de leurs Sindicats. Elles n'ont pa; eu, jusqu'ici, satisfaction, Ies lexles relatils à la taxe de luxe ne permettont pas d'élablir de distinctions entro Jos fall do peche sclon le caractère de leur exploitation.

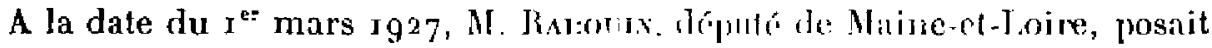
i M. le Ministre des Finances la question ricrile suivanle :

"L'Administration de l'Furegistrenent est.ell. fondéc à eviger le paiement de la taxe de $12 \%$ (laxe de luxe) instituéc sur les Jocalions du droit de pèche et de chasse par l'arlicle rg de la loi du ir juillet r9?o (modifié p.r l'article 86 de la loi du 13 juillel $r(g)$ a

" $1^{\circ}$ Les locations de terrains nì on áleve lev volnilles (avirulture) on les lapins (cuniculiculture);

" $2^{\circ}$ Les locations de fonds d'eau cì on fileve des poissons (pisciculture) ?

" 1 ! est spécifié que, dans chncun des cas emiangés ci-dessus, il sagit d'encios au sens légal du terme, c'est-it-dire dans lesquels les animaux sont la propriété de celui qui les elève .

Ja réponse ci-après fut faite à la rate du ag nars iga7:

"La taxe édictée par larticle sn de la loi du is juillet rgzo ne frappe que la location de droits de pêchc ct re droits de chasse. Ia question de 

savoir si les contrats envisagés par I'honorable député donnent ouverture à la taxe ne peut ètre rísolue qu'au vu dos actes et en pleine connaissance des circonstances particulières à chaque afiaire ".

D'un autre côté, la Justice a été saisic.

A Ia suite de la réclamation. par l'Administration de l'Enregistrement, de la taxe de luxe à denx fermiers d'élangrs de la Ilaule-Sonme, te Trihunal civil de Péronne, devint lequel les inléressés avaient fait opposition à la contrainte décernéc contre eux, Jeur donna gain de cause.

L'Administration de l'Fnregristrement s'inclina pour l'une des espèces, le fermier étant locataire de l'immeable lui-même.

Par contre, elle se pourvut on Cassation alins le second cas, dans lequel le bail spécifiajt que la jouissance du prencur porlajt sur le "droit de picche "d'un étang et non sur l'élingr lui-mine.

La Cour supreme, par son arrêl du 27 ortobre $19 ? 7$, a apprécié comme il suit le caractire de la taxe imposíc aux losabires de la fiche (ou de la chasse) par la loi du 3 i juillet ig?o.

La taxe est déclaréc applicable an " poiscomice ", attendu que.le texte de la loi n'autorise amrune distiuction cutro le iocatoirc gui recherche dans la pêche un simple ą̧rémont cl colıi qui veut entreprendro une exploitation lurrative; c'est à tort que renle tave pest qualitiéce "de luxe et de plaisir $\%$.

Il n'en serait autrement que s'il s'a risiajil ane location de l'immeuble permettant de réaliser dautres profits que: la pèche; ainsi, pour un étang, la coupe de taillis, de joncs, de roseaux.

Cette distinction peut parâtre sulfile, mais elle est fondéc sur le texte de la loi qui excepte do la taxe les loratairs des immeubles sur lesquels s'exerce le droit rle piche (ou de chasse). A isısi les carpiculteurs qui veulent se livrer à leur indsıtric sur dos fonds dliul appartenant à antrui, doivent rédiger le bail en stipulant. qli it: cot:melsul lower les fouds purement $\mathrm{et}$ simplement, et non pas sevtement he durit de pielie sur res fonds. Ils pourront "filement préciser êils useroul du fomd, non uniquement pour l'exploitation piscicole, mais nussi pour l'xploitation de tous autres produits donl ce fond est susceptible, ainsi par la culture de l'assec, eto.'

Fant d-mné l'interpretation de his Coar de cassation, le pisciculteur qui ne prendrait pas ces précaulions ur scrit pas assure d'echapper à la tixe tant gue la loi de rg\%o n'a tion lelle que la suivante:

"Cette laxe est exclusivement applirable aux localaires qui usent de leur droit pour le sport el ron poux une exploitation piscicole ". 